\title{
Decreased expression of miR-146a and miR-155 contributes to an abnormal Treg phenotype in patients with rheumatoid arthritis
}

\author{
Qihui Zhou, Sonja Haupt, Johannes T Kreuzer, Ariane Hammitzsch, Fabian Proft, \\ Carla Neumann, Jan Leipe, Matthias Witt, Hendrik Schulze-Koops, Alla Skapenko
}

\begin{abstract}
Handling editor Tore K Kvien
- Additional material is published online only. To view please visit the journal online (http://dx.doi.org/10.1136/ annrheumdis-2013-204377).

Division of Rheumatology and Clinical Immunology, Medizinische Klinik und Poliklinik IV, University of Munich, Munich, Germany

Correspondence to Dr Alla Skapenko, Division of Rheumatology and Clinical Immunology, Medizinische Klinik und Poliklinik IV, University of Munich, Pettenkoferstr. 8a, 80336 Munich, Germany: alla.skapenko@med. uni-muenchen.de
\end{abstract}

QZ and SH contributed equally.

Received 31 July 2013 Revised 20 January 2014 Accepted 24 January 2014 Published Online First 21 February 2014

\section{ABSTRACT}

Objectives MicroRNAs (miRNAs) have been implicated in the pathogenesis of autoimmune diseases, not least for their critical role in the regulation of regulatory $T$ cell (Treg) function. Deregulated expression of miR-146a and miR-155 has been associated with rheumatoid arthritis (RA). We therefore investigated miR-146a and miR-155 expression in Tregs of patients with RA and their possible impact on Treg function and disease activity. Methods Expression of miR-146a and miR-155 was assessed in RA patients and controls. MiRNA expression was correlated with disease activity and expression of target genes. Interference with biological activity of miRNAs was evaluated in functional Treg assays.

Results Diminished upregulation of miR-146a and miR-155 in response to $T$ cell stimulation was found in Tregs of RA patients. Diminution of miR-146a expression was observed in particular in patients with active disease, and correlated with joint inflammation. In patients with active RA, Tregs demonstrated a proinflammatory phenotype characterised by inflammatory cytokine expression. This was due to an augmented expression and activation of signal transducer and activator transcription 1 (STAT1), a direct target of miR146a.

Conclusions Our results suggest that in RA miR-146a facilitates a pro-inflammatory phenotype of Tregs via increased STAT1 activation, and contributes thereby to RA pathogenesis.

\section{INTRODUCTION}

Rheumatoid arthritis (RA) is a chronic autoimmune diseases characterised by synovial inflammation and a progressive cell-mediated destruction of joints. ${ }^{1}$ Regulatory CD25 positive CD4 T cells (Tregs) expressing Foxp3 control the onset and the development of autoimmune disease. Their depletion in mice results in spontaneous development of several autoimmune diseases like, for example, polyarthritis. ${ }^{2}$ Although in autoimmune diseases, Tregs are present in normal frequency and numbers in the periphery and are even enriched at local sites of inflammation like the synovium in RA, their suppressive capacity seems to be diminished. ${ }^{34}$ On the one hand, the pro-inflammatory milieu characteristic for ongoing autoimmune inflammation, in particular cytokines like tumour necrosis factor (TNF), IL-6, or IL-21, might attenuate Treg suppressive capacity. ${ }^{5-8}$ On the other hand, distinct intrinsic factors, such as genetic factors, may also contribute to a diminished suppressive phenotype of Tregs in RA. For instance, functional polymorphisms in the Foxp3 gene have been reported to be associated with susceptibility to several autoimmune diseases. ${ }^{9-11}$ Moreover, miRNAs have recently been shown to play a critical role in the regulation of the immune function of Tregs as Treg-specific ablation of either one of the two key miRNA-processing enzymes, Dicer or Drosha, results in severe autoimmunity in mice. ${ }^{12} 13$

MiRNAs are about 23nt small non-coding RNAs that regulate mRNA expression at the posttranscriptional level by directing mRNA degradation or transcriptional repression. ${ }^{14}$ Alterations in miRNA expression levels impact expression of the target genes in a 1.2-fold to 4-fold manner, and represent, therefore, a fine-tuning mechanism regulating protein expression. ${ }^{15}$ In RA, altered expression, in particular of two miRNAs, miR-146a and miR-155, has repeatedly been reported to be associated with the disease. Elevated levels of miR-146a and miR-155 were observed in synovial fibroblasts from RA patients as well as in RA synovial tissue. $^{16-18}$ Alterations in the miR-146a expression profile were found in total peripheral mononuclear as well as in CD4 T cells. ${ }^{19}{ }^{20}$ Analysis of the miRNAs in an experimental disease model revealed that treatment of mice with miR-146a in the course of collagen-induced arthritis (CIA) prevented joint destruction, ${ }^{21}$ whereas miR-155-deficient mice were completely resistant to the development of CIA. ${ }^{18}$ To understand the possible impact of miRNAs on Treg function in RA, we sought to analyse whether expression of miR-146a and miR-155 is altered also in Tregs of RA patients. We observed miR-146a alterations in Tregs and miR-155 alterations in CD4 T cells. Alterations in miR-146a but not miR-155 expression correlated with disease activity. Moreover, it correlated with augmented expression and activation of signal transducer and activator of transcription 1 (STAT1), a direct target of miR-146a, and lead likely to a pro-inflammatory Treg phenotype suggesting an essential role of miR-146a in regulation of suppressor Treg function in RA, and thereby, in RA pathogenesis.

\section{MATERIALS AND METHODS \\ Study population}

Peripheral blood was obtained from 61 patients with RA, and 49 age-matched and gender-matched 
Table 1 Clinical and demographic characteristics of the study population*

\begin{tabular}{lll}
\hline & RA patients $(\mathbf{n}=61)$ & Healthy controls $(\mathbf{n}=\mathbf{4 9})$ \\
\hline Age, years & $56.7 \pm 13.1$ & $50.1 \pm 8.3$ \\
Female/male (n) & $48 / 13$ & $36 / 13$ \\
Disease duration, years & $6.7 \pm 9.0$ & N.A. $\dagger$ \\
RF positive, \% & 80.3 & N.D. \\
Anti-CCP positive, \% & 80.3 & N.D. \\
DAS28 & $3.8 \pm 1.5$ & N.A. \\
TJC28 (n) & $4.6 \pm 5.3$ & N.A. \\
SJC28, (n) & $4.5 \pm 5.1$ & N.A. \\
CRP, mg/dL & $1.0 \pm 2.0$ & N.D. \\
ESR, mm/h & $13.9 \pm 12.4$ & N.D. \\
\hline
\end{tabular}

${ }^{*}$ Data are shown as mean \pm SD or absolute numbers.

†N.A., not applicable.

$¥ N$.D., not determined.

Anti-CCP, anti-cyclic citrullinated peptide; CRP, C-reactive protein; DAS28, Disease

Activity Score in 28 joints; ESR, erythrocyte sedimentation rate; $R A$, rheumatoid

arthritis; RF, rheumatoid factor; SJC28, swollen joint count on 28 joints; TJC28, tender joint count on 28 joints.

healthy individuals (table 1). Patients fulfilled the 2010 EULAR/ American College of Rheumatology (ACR), or the ACR 1987 criteria for RA. ${ }^{22}{ }^{23}$ Demographic and clinical parameters, such as age, gender, disease duration, tender joint count on 28 and on 68 joints (TJC28 and TJC68, respectively), swollen joint count on 28 and on 66 joints (SJC28 and SJC66, respectively), rheumatoid factor, anticyclic citrullinated peptide antibodies (Ab), disease activity score in 28 joints (DAS28), C-reactive protein (CRP), and erythrocyte sedimentation rate (ESR) were collected at the time of blood sampling. At the study entree 23/61 of patients were untreated, 17/61 were on steroids either alone or in combination with disease modifying anti-rheumatic drugs (DMARDs) and/or biologics, 35/61 were treated with DMARDs (methotrexate or leflunomide) either alone or in combination with prednisolone and/or biologics, and 19/61 were receiving biologics alone or in combination (TNF inhibitors, tocilizumab, rituximab, or abatacept). All subjects provided written informed consent. The study was approved by the Ethics Committee of the Hospital of the University of Munich.

\section{T cell purification and culture}

Tregs and CD25 negative CD4 T cells were purified from human PBMCs by a human CD4-CD25 regulatory $\mathrm{T}$ cell isolation kit (Miltenyi Biotec, Bergisch Gladbach, Germany). The purity of Tregs and CD25 negative CD4 T cells was controlled by flow cytometry using monoclonal Abs to CD3, CD4, CD25, and CD127. Typically, $\geq 95 \%$ of isolated cell populations were CD3/CD4 positive. Tregs were $\geq 90 \%$ CD25 positive and $\sim 80 \%$ CD127 negative. CD25 negative CD4 T cells were $\geq 98 \%$ negative for CD25 and $\geq 98 \%$ positive for CD127. The purity and number of the isolated cell populations were comparable between patients and controls (online supplementary figure S1). T cells were stimulated with anti-CD3/ CD28-coated beads at a 1:1 cell-to-bead ratio (Dynabeads, Life Technologies, Carlsbad, California, USA) in the presence of 100 U/mL IL-2 (Chiron, Emeryville, California, USA) in Roswell Park Memorial Institute (RPMI) 1640 supplemented with $50 \mathrm{U} / \mathrm{mL}$ penicillin $\mathrm{G}, 50 \mu \mathrm{g} / \mathrm{mL}$ streptomycin, $2 \mathrm{mM}$ L-glutamine (all from Life Technologies) and 10\% normal human serum.

\section{RNA extraction and cDNA synthesis}

Total RNA was extracted from $\mathrm{T}$ cells using the RNeasy Plus Mini kit (Qiagen, Hilden, Germany) following the manufacturer's instructions. 100 to 250 ng RNA were reversely transcribed into cDNA using AffinityScript QPCR cDNA Synthesis Kit (Agilent Technologies, La Jolla, California, USA) according to the manufacturer's instructions.

\section{Real-time PCR analysis}

MiR-146a and miR-155 were detected using TaqMan MicroRNA assays (Life Technologies). RNU48 was used as an endogenous cellular short RNA control. MRNA levels were detected using TaqMan Gene Expression Assays (Life Technologies). $\beta$-actin was used as an endogenous control. PCRs were performed in duplicates on 7500 Fast Real-Time PCR System (Life Technologies). Relative quantification was performed based on differences in cross-threshold values $(\Delta \mathrm{Ct})$ between the gene of interest and the endogenous control (ie, $\mathrm{Ct}$ (gene of interest)-Ct (endogenous control)). Relative expression of the gene of interest was calculated according to the formula $2^{-\Delta \mathrm{Ct}}$. To compare expression levels in RA patients and healthy controls, threshold value was set to a constant number for each set of experiments.

\section{Luciferase assay}

1.2 Mio Tregs were transfected with $5 \mu \mathrm{g}$ nuclear factor $\kappa$-light-chain-enhancer of activated B cells (NF- $\kappa$ B) luciferase reporter vector (pGL4.32) and $0.5 \mu \mathrm{g}$ pRL-TK, a renilla luciferase control reporter vector, (both from Promega) using an Amaxa human $\mathrm{T}$ cell Nucleofector kit (Lonza, Cologne, Germany). T cells were distributed into 9 wells of a 96-well plate $(100.000$ cell/well), and stimulated with either Dynabeads in the presence of $50 \mathrm{U} / \mathrm{mL}$ IL-2, or with $10 \mathrm{ng} / \mathrm{mL}$ phorbol 12-myristat 13-acetat (PMA) (Sigma-Aldrich) and $1 \mu \mathrm{M}$ ionomycin (Merck, Darmstadt, Germany), or left non-stimulated; $17 \mathrm{~h}$ after transfection, luciferase activity was measured using a Dual-Glo luciferase assay (Promega).

\section{Transfection}

Tregs were transfected with $1 \mu \mathrm{M}$ miRNA mimics or antagomirs using serum-free ACCELL medium (all from Thermo Scientific, Lafayette, Colorado, USA) for $1 \mathrm{~h}$ at $37^{\circ} \mathrm{C}$. Threefold excess of RPMI-1640 medium supplemented with $1 \mu \mathrm{M}$ miRNA mimics or antagomirs were added.

\section{STAT1 staining}

Freshly isolated or transfected Tregs were stimulated for $24 \mathrm{~h}$ with anti-CD3/28-coated beads. Cells were fixed, permeabilised, and then stained with PE-labelled anti-STAT1 (1/Stat1) and Alexa Fluor 647-labelled anti-STAT1 (pS727) (K51-856) (all from BD Phosflow, San Jose, California, USA).

\section{ELISA}

Freshly isolated, or transfected Tregs, were stimulated for $24 \mathrm{~h}$ with anti-CD3/28-coated beads. Levels of IFN $\gamma$, TNF and IL-17 in cell culture supernatants were assessed using Quantikine ELISA according to the manufacturer's instructions (R\&D Systems, San Diego, California, USA).

\section{T cell suppression assays}

CD25 negative CD4 T cells were labelled with $10 \mu \mathrm{M}$ carboxyfluorescein succinimidyl ester (CFSE) (Life Technologies). MiRNA mimics- or antagomir-transfected Tregs were cultured with CFSE-labelled CD25 negative CD4 T cells in a 1:1 ratio in the presence of soluble anti-CD3 and feeder cells for 4 days. Proliferation of CD25 negative CD4 T cells was assessed by flow cytometry based on CFSE dilution. 


\section{Statistical analyses}

Statistical analysis was performed using Prism 5.0 Software for Macintosh (GraphPad Software, San Diego, California, USA). Normal distribution was controlled by Kolmogorov-Smirnov. Differences between study cohorts were determined using Student t test. Spearman's rank correlation coefficient was performed to test statistical dependency between two variables; $\mathrm{p}<0.05$ was considered statistically significant.

\section{RESULTS}

\section{Diminished expression levels of miR-146a and miR-155 in Tregs from RA}

Analysis of miR-146a and miR-155 expression in freshly isolated Tregs and CD25 negative CD4 T cells revealed that both miRNAs were expressed at comparable levels in both cell populations. There were no differences in the expression levels between the analysed RA patient cohort and healthy controls (figure 1A,B).

In response to anti-CD3/CD28 stimulation, expression levels of miR-146a decreased slightly in Tregs, whereas in CD25 negative CD4 T cells they were markedly downregulated (figure 1A). Important was the observation that the expression of miR-146a in Tregs but not in CD25 negative CD4 T cells was significantly lower in RA patients than in healthy controls 1 and 2 days after stimulation. Expression levels of miR-146a in Tregs of RA patients upon $\mathrm{T}$ cell receptor (TCR) stimulation accounted for about $70 \%$ of the expression levels in healthy individuals (see online supplementary table S1).

By contrast, expression of miR-155 was highly upregulated in response to anti-CD3/CD28 in Tregs and CD25 negative CD4 T cells (figure 1B). Still similar to the expression of miR-146a, expression of miR-155 was significantly lower in RA patients than in controls 1 and 2 days after stimulation. The diminished miR-155 expression in RA seemed, however, to be a rather general characteristic of CD4 T cells and not of Tregs in particular as it was observed in both $\mathrm{T}$ cell populations, Tregs and CD25 negative CD4 T cells. Yet, in Tregs of RA patients, expression levels of miR-155 upon $\mathrm{T}$ cell receptor (TCR) stimulation accounted for about $50 \%$ of the expression levels in healthy individuals (see online supplementary table S1).

\section{Different expression levels of miR-146a but not of miR-155 in RA in patient groups stratified based on disease activity}

When we stratified the patients based on disease activity into patients with active disease (DAS28 >3.2) and patients with low disease activity (DAS28 $\leq 3.2$ ), we observed a marked difference in the expression of miR-146a in Tregs (figure 1C). Whereas Tregs from patients with low disease activity expressed high levels of miR-146a comparable with that of healthy controls, Tregs from patients with active disease expressed significantly lower levels of the miRNA. Tregs from patients with active RA expressed around 1.5-fold to 2-fold lower levels of miR-146a as compared to healthy controls or patients with low disease activity (see online supplementary table S2). No such differences between patient groups and healthy controls were observed in CD25 negative CD4 T cells.

Analysis of miR-155 expression revealed that upregulation of the miRNA after TCR stimulation in Tregs was less pronounced in both RA patient groups than in healthy individuals (figure 1D). Expression levels of the miRNA were around three-fold lower in RA Tregs than in healthy controls without a difference between the patient groups (see online supplementary table S2). A similar trend was observed in CD25 negative CD4 T cells.
Expression levels of miR-146a correlate with clinical parameters of disease activity

Because of the Treg-characteristic expression pattern of miR-146a in RA, we next carried out a correlation analysis between clinical parameters and expression levels of miR-146a in Tregs (figure 2). Indeed, there was an inverse correlation between DAS28 and the expression levels of miR146a (figure 2A). Also, SJC28, SJC66 and TJC28, TJC68 correlated negatively with miR-146a expression (figure 2B,C). By contrast, there were no such apparent correlations between CRP or ESR and miR-146a expression expression (figure 2D,E). This confirms our observation that in RA, expression of miR-146a levels in Treg correlates with disease activity.

\section{MiR-146a and miR-155 expression levels in RA do not correlate with expression of their target genes involved in NF-кB signalling}

Both miRNAs, miR-146a and miR-155, have been reported to be involved in a negative feedback loop regulating the NF- $\mathrm{B}$ signalling pathway. ${ }^{24} \mathrm{We}$, therefore, next investigated expression of their target genes within the NF- $\mathrm{KB}$ activation pathway in Tregs from RA patients with active disease and with low disease activity (figure 3). MiR-146a has been reported to regulate expression of interleukin-1 receptor-associated kinase 1 (IRAK1) and TNF receptor associated factor 6 (TRAF6), ${ }^{25}$ whereas miR-155 targets I- $\kappa$ B kinase epsilon (IKKe). ${ }^{26}$ Expression of the IRAK1 mRNA was upregulated in response to anti-CD3/ CD28 stimulation (figure $3 \mathrm{~A}$ ). By contrast, expression of TRAF6 and IKKe was rather down-regulated (figure 3B,C). Interestingly, expression levels of these three genes were generally higher in RA patients as compared with healthy controls independent of disease activity. NF- $\kappa \mathrm{B}$ activity was, however, comparable between patients and controls (figure 3D). No correlation was, therefore, observed between the expression levels of miR-146a or miR-155 and the NF- $\kappa B$ signalling pathway (online supplementary figures S2A,B, 3A).

\section{Correlation of miR-146a expression levels with expression of STAT1 but not of miR-155 with suppressor of cytokine signalling 1 (SOCS1)}

When we analysed the expression of two other genes reported to be regulated by miR-146a and miR-155 in particular in Tregs, STAT1 and SOCS1, ${ }^{27-29}$ we observed a striking upregulation of their mRNA expression in response to TCR stimulation from Tregs in patients with active disease but not in healthy controls, and not in the RA patient group with low disease activity (figure 4A). The upregulation of STAT1 mRNA in the active group of patients but not in patients with low disease activity correlated well with the downregulation of miR-146 expression in Tregs in patients with active disease but not with low disease activity (figure 1C, supplementary figure S2C). Moreover, higher STAT1 protein expression and phosphorylation in response to stimulation in patients with active disease was observed (figure 4B).

Expression of SOCS1 (figure 4A), a target of miR-155, in Tregs did not correspond with the expression pattern of miR-155 shown in figure 1D (see online supplementry figure $\mathrm{S} 3 \mathrm{C})$. Whereas the active RA group demonstrated an increase in SOCS1 expression and a simultaneous down-regulation of miR-155, patients with low disease activity showed diminished expression of both (figures 1D, 4A), indicating an additional mechanism controlling SOCS1 expression. 
A miR-146a

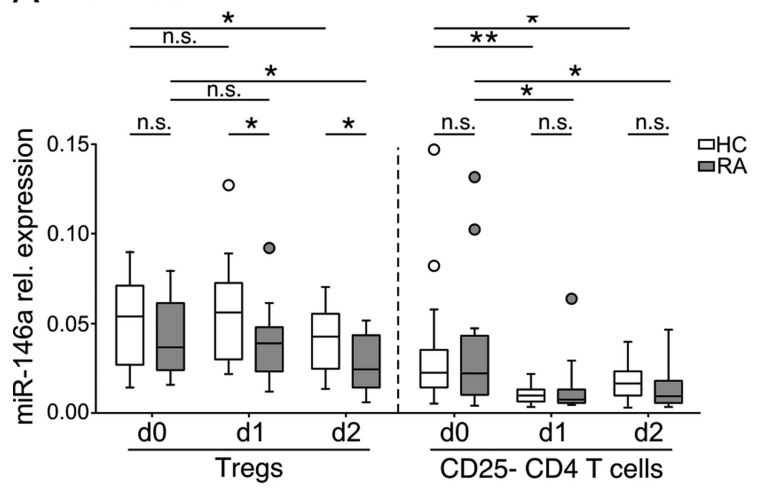

B $\operatorname{miR}-155$

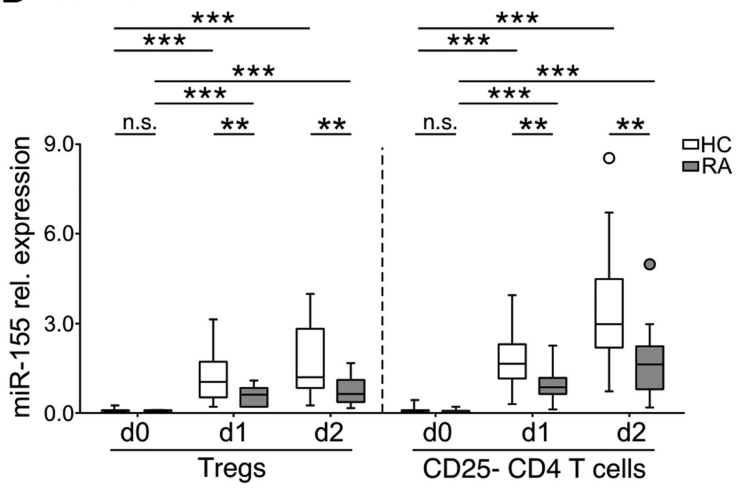

C miR-146a

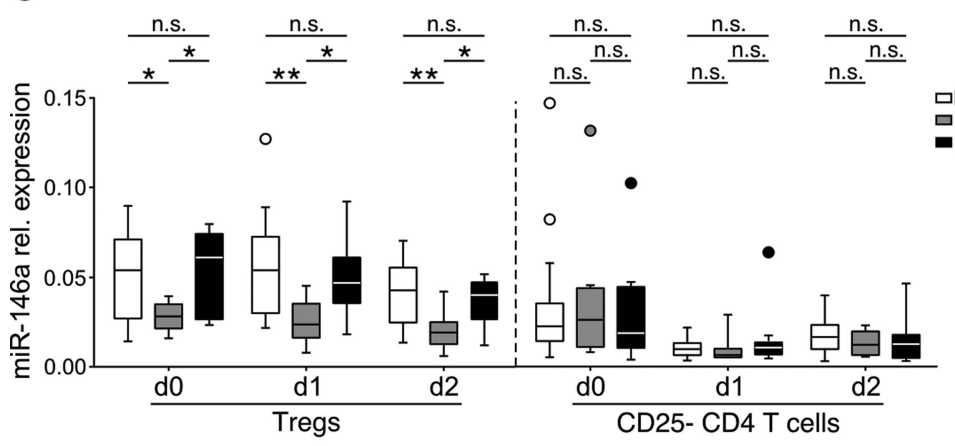

口HC

DRA DAS $28>3.2$

-RA DAS $28 \leq 3.2$

D miR-155

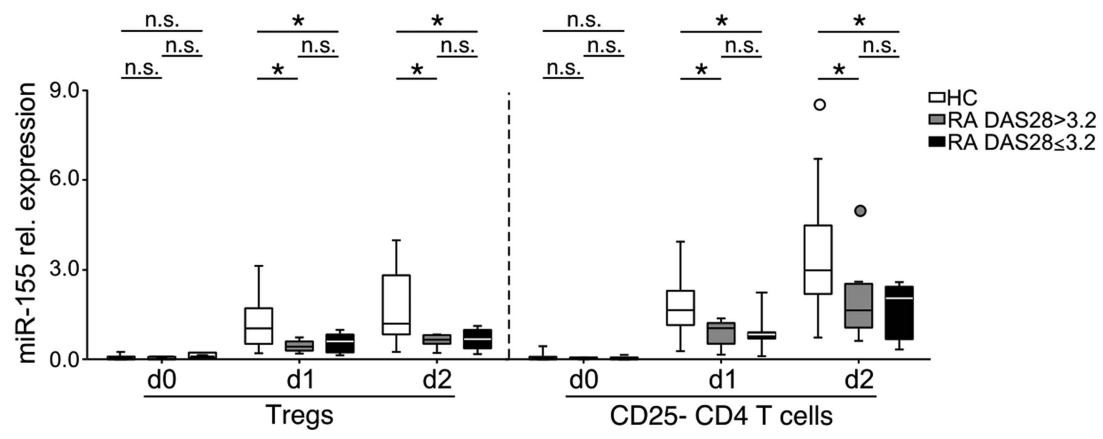

Figure 1 MiR-146a and miR-155 expression levels in Tregs and CD25 negative CD4 T cells. Tregs and CD25 negative CD4 T cells were isolated and stimulated with anti-CD3/CD28 beads for a total of 2 days. MiRNA expression was assessed in freshly isolated (d0) and in stimulated cells at $24 \mathrm{~h}(\mathrm{~d} 1)$ and $48 \mathrm{~h}(\mathrm{~d} 2)$ by real-time PCR using TaqMan gene expression assays. MiRNA expression in relation to expression levels of RNU48 is demonstrated. Expression levels of miR-146a (A) and of miR-155 (B) in 24 healthy controls (HC) and 19 patients with rheumatoid arthritis (RA). (C, D) Expression of miR-146a (C) and miR-155 (D) in $24 \mathrm{HC}$ and in RA patient stratified according to disease activity: patients with active disease-RA DAS28 $>3.2(n=8)$ and patients with low disease activity-RA DAS28 $\leq 3.2(n=11)$. Data are shown as box plots demonstrating minimum, maximum, median and 25 th and 75 th percentiles. ${ }^{*} p<0.05,{ }^{* *} p<0.01,{ }^{* *} p<0.001$ by Student $t$ test.

\section{Altered cytokine secretion pattern in Tregs from patients with active RA}

Alterations in the expression of miR-146a or miR-155, and subsequently of STAT1 and SOCS1, might modify cytokine secretion profiles of CD4 T cells. ${ }^{27} 3031$ We, therefore, next analysed expression of the pro-inflammatory cytokines, IFN $\gamma$, TNF, IL-17 and IL-2, in Tregs from RA patients stratified again according to their disease activity (figure 4C). In response to anti-CD3/CD28 stimulation, all cytokines were upregulated in Tregs at mRNA level within $24 \mathrm{~h}$ and downregulated after $48 \mathrm{~h}$. Strikingly, however, upregulation of cytokine expression was more pronounced in Tregs from RA patients with active disease than in healthy controls or in patients with low disease activity. Patients with low disease activity expressed even diminished levels of the pro-inflammatory cytokines in comparison with healthy controls. The increase of cytokine mRNA after $24 \mathrm{~h}$ of stimulation in Tregs of patients with active disease was reflected by cytokine concentrations in cell culture supernatants (figure 4D). These data together indicate that in RA, changes in miR-146a or miR-155 expression might indeed lead to alterations in the Treg phenotype.

Cytokine secretion pattern, but not the suppressive capacity of Tregs correlates with miR-146 and miR-155 expression

To verify this hypothesis we next analysed cytokine expression in Tregs treated with either mimics or antagomirs of miR-146a and miR-155 (figure 5A). Indeed, treatment with mimics of miR-146a 

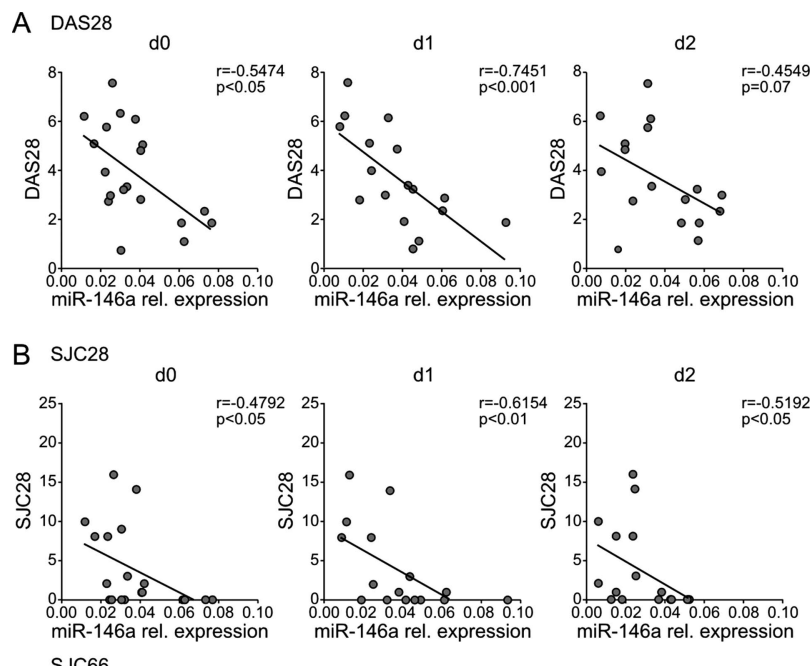

C TJC28
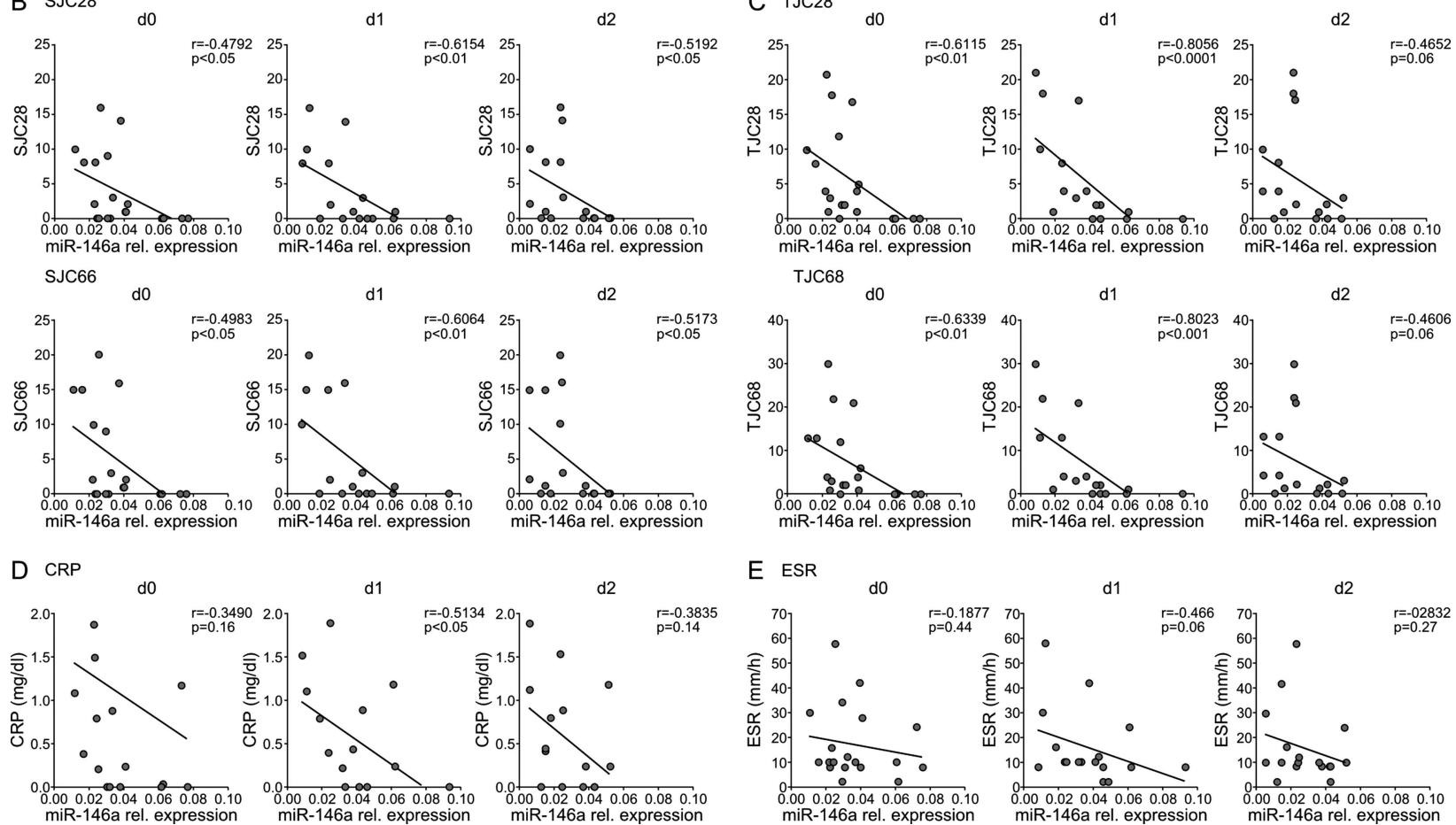

E ESR
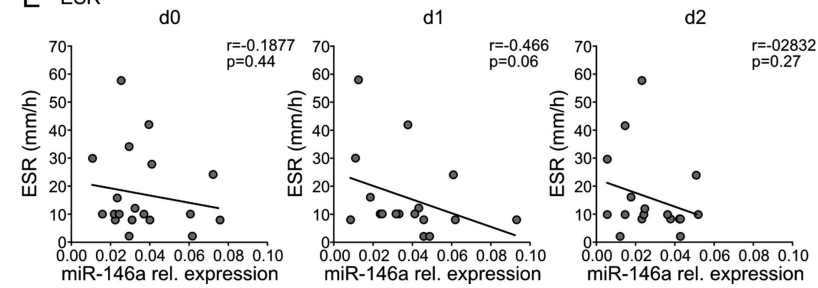

Figure 2 Correlation between clinical parameters and the expression levels of miR-146a in Tregs in rheumatoid arthritis (RA). Tregs were isolated and stimulated with anti-CD3/CD28 beads for a total of 2 days. MiRNA expression was assessed in freshly isolated (d0) and in stimulated cells at $24 \mathrm{~h}(\mathrm{~d} 1)$ and $48 \mathrm{~h} \mathrm{(d2)} \mathrm{by} \mathrm{real-time} \mathrm{PCR} \mathrm{using} \mathrm{TaqMan} \mathrm{gene} \mathrm{expression} \mathrm{assays.} \mathrm{MiRNA} \mathrm{expression} \mathrm{in} \mathrm{relation} \mathrm{to} \mathrm{the} \mathrm{expression} \mathrm{levels} \mathrm{of} \mathrm{RNU48} \mathrm{is}$ demonstrated. Disease activity on 28 Joints (DAS28) (A), swollen joint count on 28 joints (SJC28) and SJC66 (B), tender joint count on 28 joints (TJC28) and TJC68 (C), C-reactive protein (CRP) (D) and erythrocyte sedimentation rate (ESR) (E) were plotted against the miR-146a expression levels at $\mathrm{d} 0, \mathrm{~d} 1$ and $\mathrm{d} 2$. Correlations were determined by Spearman's rank correlation coefficient.

reduced cytokine secretion, whereas mimics of miR-155 increased the cytokine production. Neutralisation of the miRNA effects through antagomirs led to opposite results. Interestingly, however, once both miRNAs were mimicked or neutralised simultaneously, the cytokine secretion profile was rather similar to that observed during manipulation of miR-146a (figure 5A). The intervention with miR-146a led also to significant alterations in STAT1 expression and phosphorylation (figure 5B). Whereas mimicking of miR-146a diminished STAT1 expression and phosphorylation, neutralisation of the miRNA increased both. Remarkably, despite the pronounced effect on cytokine expression profile, intervention with the expression of these two miRNAs did not affect the in vitro suppressive capacity of Tregs (figure 5C).

\section{DISCUSSION}

Based on the apparent deregulation of miR-146a and miR-155 expression in RA and, moreover, their critical involvement in the onset of arthritis in animal models, we have investigated in the current study expression of miR-146a and miR-155 in Tregs, and have analysed their impact on Treg function in patients with RA.

We observed a diminished expression of both miRNAs, miR-146a and miR-155, in Tregs of patients indicating some disease-dependent abnormalities in the regulation of their expression. MiR-146a and miR-155 are widely expressed and are involved in the differentiation and activation of both, the innate and the adaptive immune systems. ${ }^{32}$ In particular, miR-146a has been initially reported to be upregulated in macrophages in response to TLR-mediated NF- $\mathrm{KB}$ signalling. ${ }^{25} 33$ MiR-146a seems to be an important player in the NF- $\mathrm{KB}$ signalling pathway in innate immunity as it downregulates the NF-KB signalling transducers, IRAK1 and TRAF6 and, thereby, calms NF- $\mathrm{kB}$ signals. Observations from miR-146a-deficient mice suggest that also in CD4 and CD8 T cells, miR-146a might be upregulated in response to TCR-mediated NF- $\mathrm{kB}$ signalling and, in turn, might downregulate NF-kB activity through repressing IRAK1 and TRAF6. ${ }^{34}$

Interestingly, however, we did not observe an upregulation of miR-146a in response to TCR stimulation, either in Tregs or in CD25 negative CD4 $\mathrm{T}$ cells, despite NF-kB-activation that occurred in response to our stimulatory conditions (figures 1A, $3 \mathrm{D})$. The human origin of the cells in our study allows us, therefore, to suggest different signalling pathways downstream of TCR signalling in CD4 $\mathrm{T}$ cells in humans than in mice that result in miR-146a expression. Moreover, evidence suggests that 
A IRAK1 mRNA

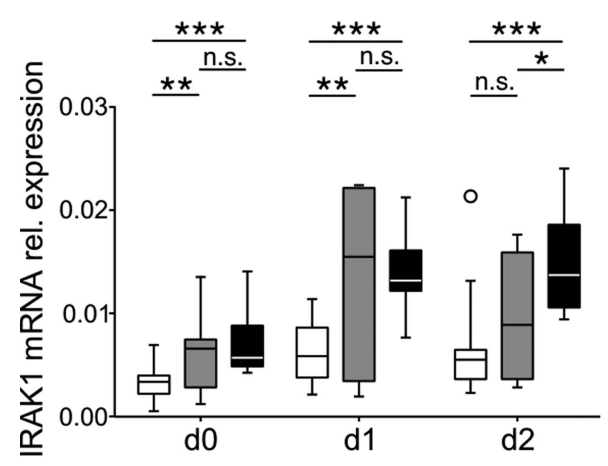

C IKKE mRNA

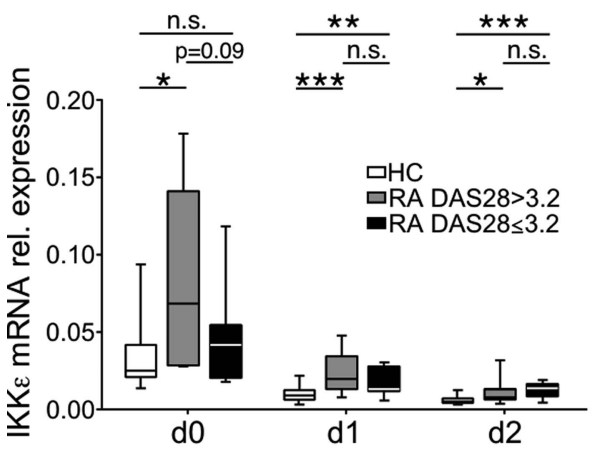

\section{B TRAF6 mRNA}

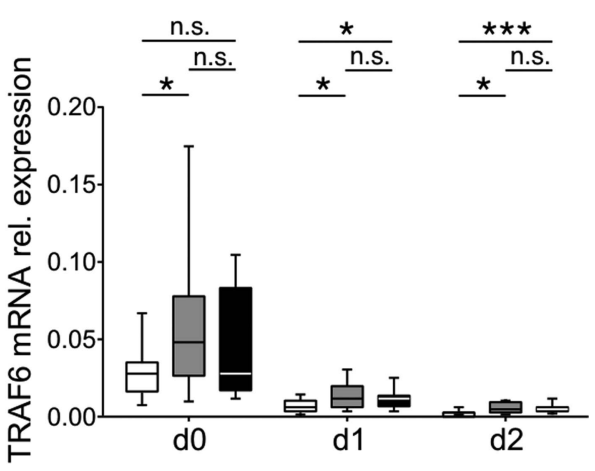

D NF-kB activity

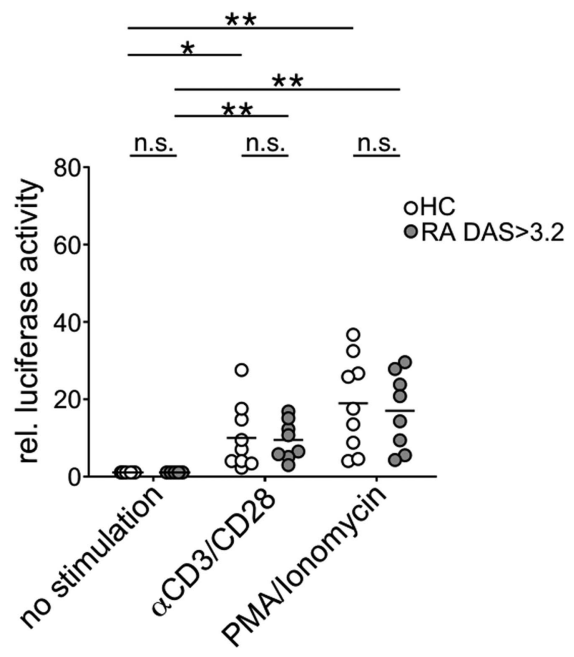

Figure 3 Analysis of NF- $\mathrm{KB}$ signalling pathway. (A-C) Tregs were isolated and stimulated with anti-CD3/CD28 beads for a total of 2 days. MRNA expression was assessed in freshly isolated (d0) and in stimulated cells at $24 \mathrm{~h}(\mathrm{~d} 1)$ and $48 \mathrm{~h}(\mathrm{~d} 2)$ by real-time PCR using TaqMan gene expression assays. Relative mRNA expression normalised to the expression levels of B-actin mRNA is demonstrated. Expression levels of IRAK1 (A), TRAF6 (B) and IKKE (C) in 24 healthy controls and in rheumatoid arthritis (RA) patients grouped according to disease activity: active RA-DAS28 $>3.2$ ( $n=8$ ) and low disease activity-DAS28 $\leq 3.2(\mathrm{n}=11)$, are shown as box plots demonstrating minimum, maximum, median and 25 th and 75 th percentiles. (D) Isolated Tregs were transfected with an NF- $\mathrm{B}$ reporter vector and stimulated either with anti-CD3/CD28 beads or with phorbol 12-myristat 13-acetat (PMA) and ionomycin. Luciferase activity was analysed $17 \mathrm{~h}$ later. Relative luciferase activity from 9 HC and 8 RA patients is shown. ${ }^{*} \mathrm{p}<0.05,{ }^{* *} \mathrm{p}<0.01,{ }^{* *} \mathrm{p}<0.001$ by Student $t$ test.

changes in the $\mathrm{T}$ cell phenotype in response to miR-146a deletion might occur not necessarily only as a result of NF- $\mathrm{NB}$ signalling but also because of augmented expression of other target genes of miR-146a, like for example, STAT1. ${ }^{27}$ In our study, we indeed observed a marked correlation between miR-146a and STAT1 expression, but not between miR-146a and IRAK1 or TRAF6 expression (online supplementary figure S2). IRAK1 and TRAF6 mRNAs were upregulated in patients with active and non-active disease, while miR-146a expression was downregulated only in patients with active RA (figures $1 \mathrm{C}$, 3A). Putative binding sites for miR-146a can be predicted in the 3'UTR of murine and human IRAK1 and TRAF6 mRNAs. Moreover, miR-146a-mediated regulation of the expression of human IRAK1 and TRAF6 has been shown in an in vitro setting. ${ }^{25}$ Yet, it might well be that in vivo expression of these two critical molecules of the NF- $\mathrm{KB}$ signalling pathway at least in humans is subject to a complex and not only to a simplified miR-146a-mediated switch-on/off regulatory mechanism. In this regard, Pauley et $a l^{19}$ reported normal IRAK1 and TRAF6 expression in a bulk of mononuclear cells of RA patients despite the observed 2.6-fold increase in miR-146a expression.
Similar to miR-146a, miR-155 has been reported to be induced by NF- $\mathrm{NB}$ signalling. ${ }^{24}$ The result of NF- $\kappa \mathrm{B}$-induced miRNA expression, however, appears to be rather contrary between miR-146a and miR-155. Whereas miR-146a rather calms the pro-inflammatory immune response, miR-155 enhances inflammation. Deletion of miR-146a leads to a sizable proportion of IFN $\gamma$-producing CD4 $\mathrm{T}$ cells and hyperresponsiveness of macrophages to LPS. ${ }^{27}{ }^{35}$ MiR-146a-deficient mice spontaneously develop autoimmune disorders. ${ }^{35}$ MiR-155, in turn, diminishes the magnitude of the immune response in macrophages and dendritic cells. ${ }^{32}$ Mice deficient for miR-155 are biased towards Th2 differentiation and are resistant to Th17-driven autoimmune inflammation. ${ }^{18} 303136$ Interestingly, we did observe these opposite effects of both miRNAs. Tregs from active RA patients expressed on the one hand increased levels of STAT1, a target gene of miR-146a, promoting a pro-inflammatory phenotype of the cells, and on the other hand elevated levels of SOCS1, a target of miR-155, which negatively regulates inflammation (figure 4). Additionally, treatment with mimics and antagomirs led also to opposite effects on cytokine expression (figure 5A). Nevertheless, the resulting phenotype of 
A STAT1 mRNA

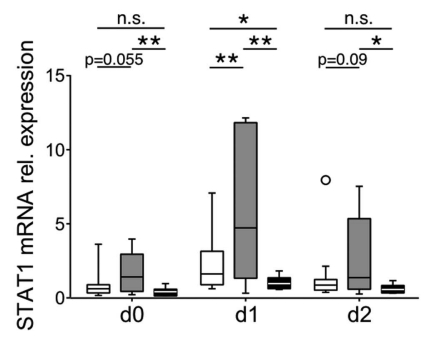

SOCS1 mRNA

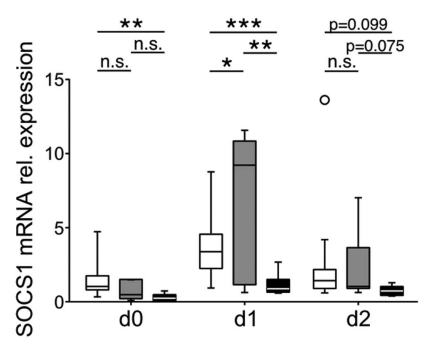

DHC DRA DAS $28>3.2$
-RA DAS $28 \leq 3.2$
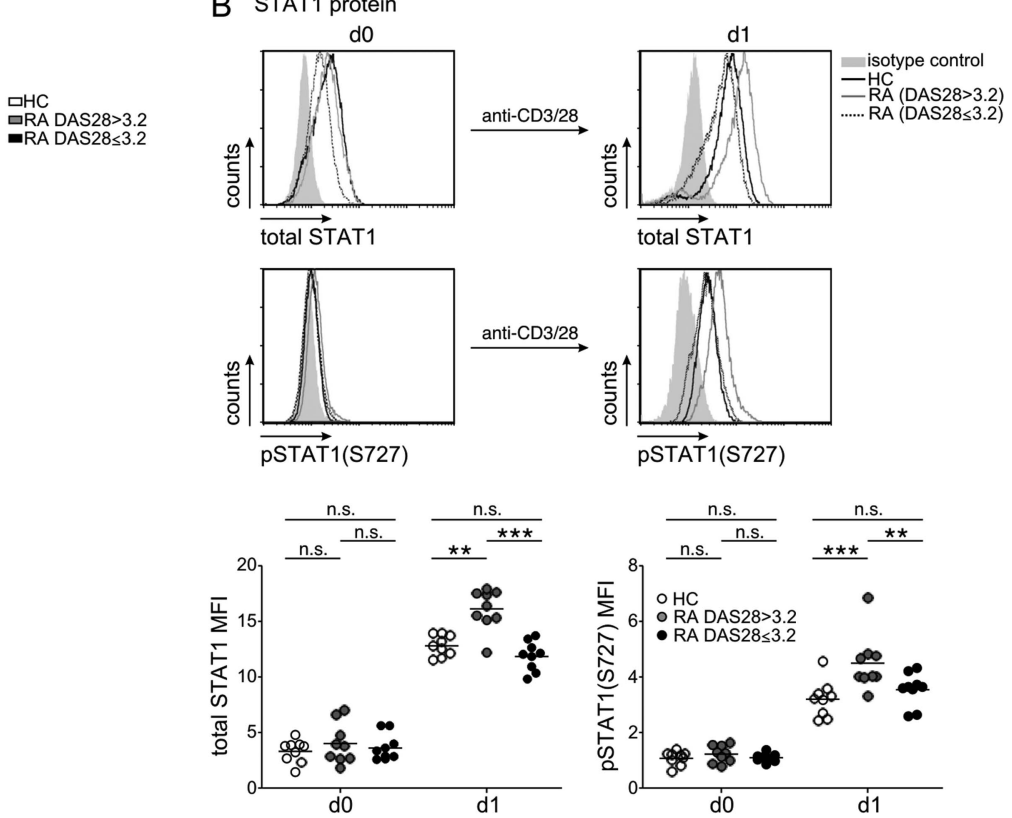
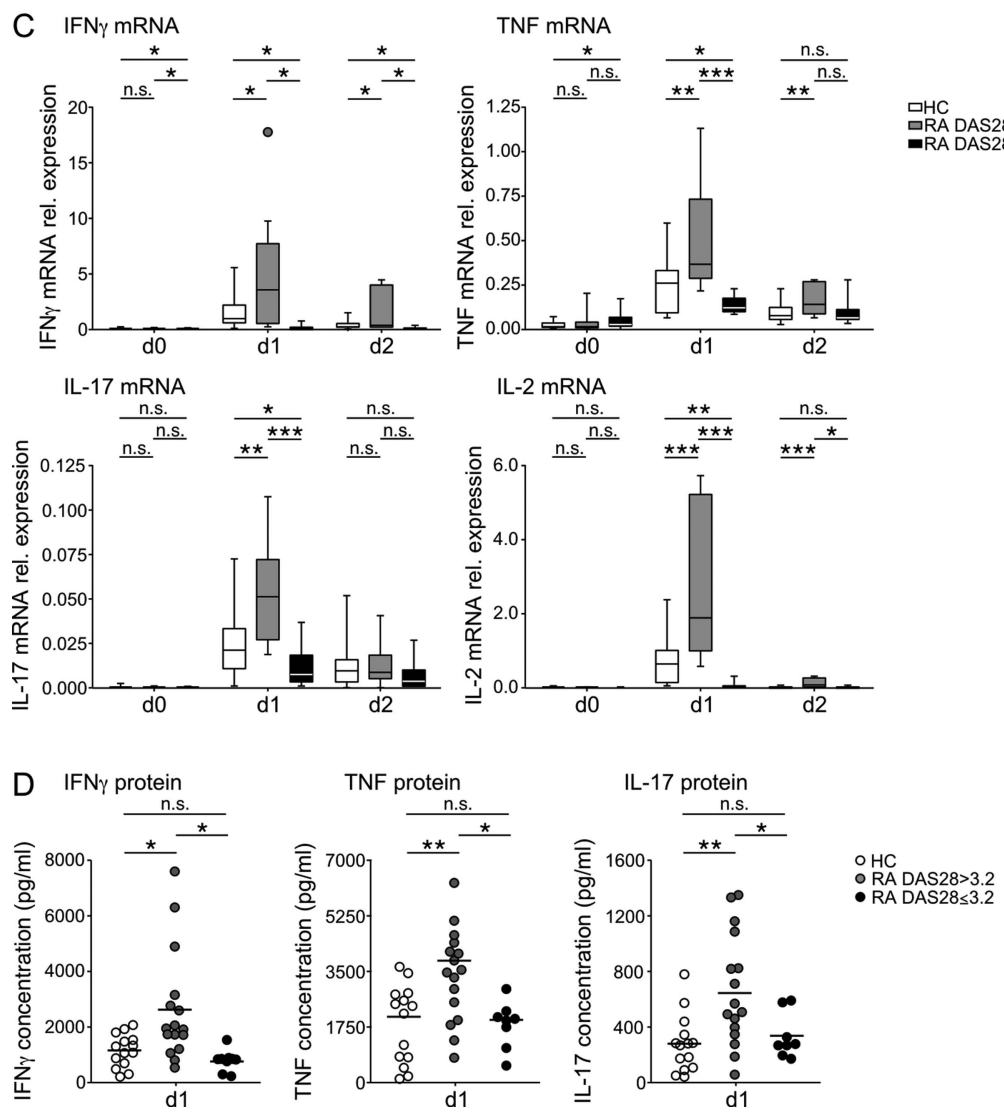

Figure 4 Analysis of STAT1, SOCS1 and cytokine expression. Tregs were isolated and stimulated with anti-CD3/CD28 beads for a total of 2 days. MRNA expression was assessed in freshly isolated (d0) and in stimulated cells at $24 \mathrm{~h}(\mathrm{~d} 1)$ and $48 \mathrm{~h}(\mathrm{~d} 2)$ by real-time PCR using TaqMan gene expression assays. Relative mRNA expression normalised to the expression levels of $B$-actin mRNA is demonstrated. Protein expression was assessed in freshly isolated (d0) and/or in $24 \mathrm{~h}$ stimulated (d1) cells by intracellular staining (STAT1) or by ELISA (cytokines). MRNA expression levels of STAT1 and SOCS1 (A) and of IL-2, TNF, IL-17 and IFN $\gamma$ (C) in 22 healthy controls (HC) and in 19 rheumatoid arthritis (RA) patients are shown as box plots demonstrating minimum, maximum, median and 25th and 75th percentiles. (B) Expression of total STAT1 protein and of phosphorylated STAT1 (pSTAT1) was assessed as mean fluorescence intensity. Representative stainings are demonstrated in the upper panel. In the lower panel results from $9 \mathrm{HC}$ and 18 RA patients are summarised. (D) TNF, IL-17 and IFN $\gamma$ at protein level were assessed in cell culture supernatants from $14 \mathrm{HC}$ and 24 RA patients $24 \mathrm{~h}$ after stimulation by ELISA. Patients were stratified according to disease activity: active RA—DAS28 $>3.2$ and low disease activityDAS28 $\leq 3.2$. ${ }^{*} p<0.05,{ }^{* *} p<0.01,{ }^{* *} p<0.001$ by Student $t$ test. 


\section{Basic and translational research}

A interference with miR-146a $(n=10)$

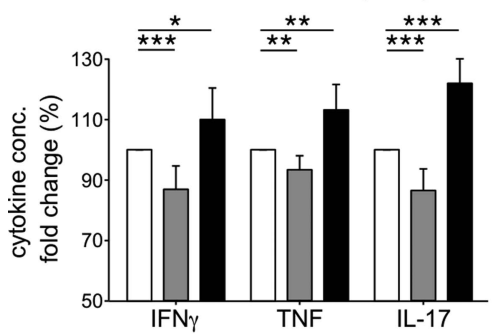

interference with miR-155 $(n=6)$

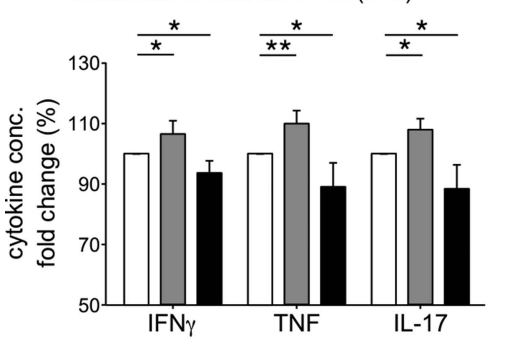

interference with miR-146a+miR-155 ( $n=3)$

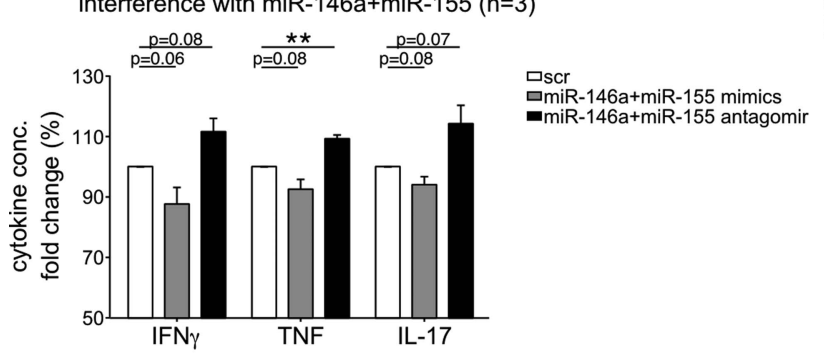

B STAT1 protein $(n=10)$

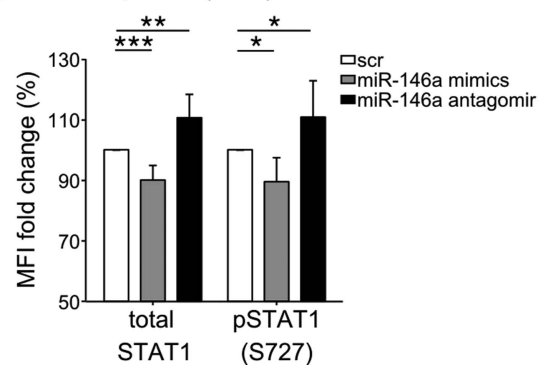

C ㅁscr DmiR-146a mimics
-miR-146a antagomir -miR-146a antagomir

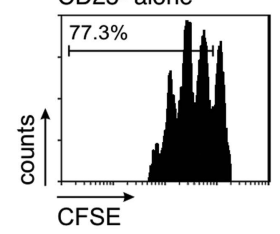

CD25- + Tregs non-treated

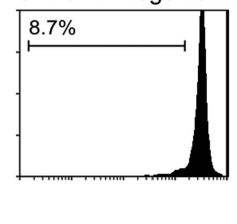

CD25- + Tregs treated with:

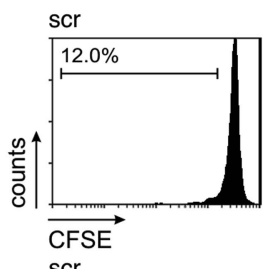

miR-146a mimics

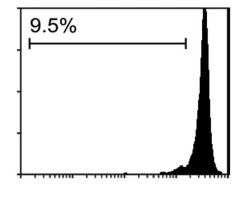

miR-146a antagomir

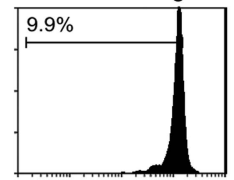

miR-155 mimics
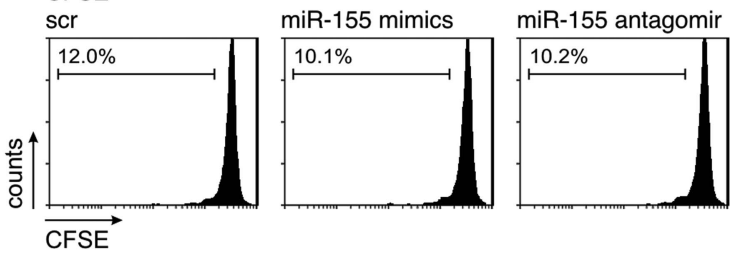

Figure 5 Effect of miR-146a and miR-155 on Treg cytokine production and suppressive capacity. Tregs were isolated, transfected with mimics or antagomirs of miR-146a or miR-155 and (A, B) stimulated with anti-CD3/CD28. $24 \mathrm{~h}$ after stimulation IFN $\gamma$, TNF and IL-17 were assessed in cell culture supernatants by ELISA (A) or expression and phosphorylation status of STAT1 (total STAT1 and pSTAT1, respectively) were assessed intracellularly based on mean fluorescence intensity (B). Data are shown as fold changes normalised to cells transfected with scrambled (scr) oligos. Mean \pm SD of 3 to 10 experiments are shown. (C) To verify the effect of intervention with miRNA activity on the suppressive capacity of Tregs,

CD25 negative CD4 T cells were isolated additionally, and labelled with CFSE. Transfected Tregs were cultured with CFSE-labelled CD25 negative CD4 T cells in a 1:1 ratio in the presence of anti-CD3 and feeder cells for 4 days. Proliferation of CD25 negative CD4 T cells was assessed by flow cytometry based on CFSE dilution. One representative experiment of three experiments is depicted. ${ }^{*} p<0.05,{ }^{* *} p<0.01,{ }^{* * *} p<0.001$ by Student $t$ test.

the Tregs was rather pro-inflammatory in patients with active disease, as expression of IL-17, TNF and IFN $\gamma$ was elevated (figure 4C,D). Thus, it appears as if the pro-inflammatory effect of reduced miR-146a expression prevails over the counteracting impact of reduced miR-155 expression, leading to pro-inflammatory alterations of the phenotype of Tregs in RA. This became obvious when we interfered with the biological activity of both miRNAs simultaneously (figure 5A). The observed diminution of miR-155 expression may, therefore, reflect an attempt of Tregs to counteract the unwanted pro-inflammatory STAT1-mediated effects by upregulation of its natural antagonist, SOCS1.
The preponderance of miR-146 over miR-155 with regard to their impact on Treg function in RA was reflected by the correlation of miR-146 but not of miR-155 with clinical parameters of disease activity. Interesting in this regard was the fact that miR-146a correlated with indicators of local joint inflammation, for example, TJC and SJC, but not with parameters suggestive of systemic inflammation, for example, CRP and ESR (figure 2). This finding suggests that miR-146a-mediated alterations in the Treg phenotype were of particular importance in synovial inflammation although it should be noted that no difference in miR-146a expression levels were detected between Tregs from the peripheral circulation and matched synovial samples (online 
supplementary figure S4). In this regard, it is interesting that diminution of miR-146a (as of miR-155) expression became only evident in response to activation indicating that the effect of these alterations strikes primarily during an ongoing immune response like, for example, local inflammation in the joint. By contrast, systemic inflammation appears to be less dependent on Treg function. In accordance with our finding, Tang et $a l^{21}$ have observed that in CIA, systemic administration of an analogue to miR-146a prevented joint destruction but failed to completely ameliorate inflammation. Likewise, in lupus, decreased levels of miR-146a in leukocytes were also associated with kidney inflammation, a prominent organ manifestation in lupus, but not with systemic inflammatory activity. ${ }^{37}$

Our data on miR-146a expression appear to be in contrast to a publication by $\mathrm{Li}$ et $a l^{20}$ reporting increased expression of miR-146a in CD4 T cells from patients with RA. The patient cohort analysed in that paper is, however, different to ours. The patients from the previous study had long-lasting disease ( $\sim 5$ years), very active RA (DAS28 6.35 \pm 1.45 ), and yet were DMARD naive. By contrast, the untreated patients included in our study had symptoms of the disease for less than 1 year $(8.2$ \pm 11.2 months) and a DAS28 of $5.02 \pm 1.13$. It might be speculated that long-lasting inflammation without any treatment in the patients analysed by Li et al might have led to secondary events influencing the miRNA expression profile that are not related to primary Treg alterations in RA. The fact that Li et al observed extremely elevated ESR $(67.94 \pm 21.19 \mathrm{~mm} / \mathrm{h})$ may support this hypothesis. On the other hand, we cannot completely exclude that the glucocorticoide or the antirheumatic therapy might at least in part have influenced results of our study. The preliminary observation that anti-TNF-treatment was associated with a change in miR-146a levels may support this notion (online supplementary figure S4). In any case, the strong correlation between miR-146a expression and disease activity independent of the therapy observed by us (figure 2) argues for a direct dependence of miRNA expression in Tregs and disease activity.

The pro-inflammatory phenotype of Tregs characterised by enhanced cytokine production in RA was also observed by others. ${ }^{38}$ Inflammatory conditions might force Tregs to become unstable and adopt a phenotype that is more characteristic of effector CD4 T cells during the evolution of an autoimmune disease. ${ }^{39}{ }^{40}$ Altered expression of miR-146a in RA Tregs might thus be the molecular correlate of such phenotypic alterations of Tregs. As reduced expression of miR-146a was particularly prominent in patients with early, treatment-naive RA patients and in patients with active disease, but not in patients with wellcontrolled or long-standing disease, our observation might argue for a role of diminished Treg function during disease onset and in disease flairs. Moreover, they might argue for a Treg control of autoimmune inflammation at rather local, organspecific sites and not at the level of systemic inflammation. Our findings indicate that a deregulated miRNA expression in Tregs might lead to harmful alterations in their phenotype and provide, therefore, novel indications for the involvement of miR-146a and miR-155 in RA pathogenesis.

Acknowledgements The authors thank Christine Schnabel for her expert technical assistance. We are thankful to Drs Grünke, Dechant, Reindl and Abahji for their help in recruiting patients. We are indebted to all patients and healthy individuals for their invaluable willingness to donate blood.

Contributors QZ and SH performed the experiments, analysed the data and wrote the manuscript. JK performed the experiments. $A H, F B, C N$, JL and $M W$ collected and analysed clinical data. HS-K designed the study and wrote the manuscript. AS designed the study, analysed the data and wrote the manuscript.
Funding This work was supported by the Deutsche Forschungsgemeinschaft (Grants SK59/4-1, Schu 786/2-5, and Schu 786/8-1), by the

Sonderforschungsbereich 571 (Autoimmunity), project D9, by the DFG Training Grant GK1202 (Oligonucleotides), project E2, and by the Verbundanträge 'ArthroMark' (projects 1 and 7, OIEC100913) and "Impam" (project 10, OIEC1008H), Federal Ministry of Education and Research of Germany. The funders had no role in study design, data collection and analysis, decision to publish, or preparation of the manuscript.

\section{Competing interests None.}

Patient consent Obtained.

Ethics approval Ethics Committee of the Hospital of the University of Munich.

Provenance and peer review Not commissioned; externally peer reviewed.

\section{REFERENCES}

1 Firestein GS. Evolving concepts of rheumatoid arthritis. Nature 2003;423:356-61.

2 Sakaguchi S, Sakaguchi N, Asano M, et al. Immunologic self-tolerance maintained by activated T cells expressing IL-2 receptor alpha-chains (CD25). Breakdown of a single mechanism of self-tolerance causes various autoimmune diseases. J Immunol 1995; 155:1151-64.

3 Jiao Z, Wang W, Jia R, et al. Accumulation of FoxP3-expressing CD4+CD25+ T cells with distinct chemokine receptors in synovial fluid of patients with active rheumatoid arthritis. Scand I Rheumatol 2007:36:428-33.

4 Lin $\mathrm{SC}$, Chen $\mathrm{KH}$, Lin $\mathrm{CH}$, et al. The quantitative analysis of peripheral blood FOXP3-expressing T cells in systemic lupus erythematosus and rheumatoid arthritis patients. Eur J Clin Invest 2007;37:987-96.

5 O'Sullivan BJ, Thomas HE, Pai S, et al. IL-1 beta breaks tolerance through expansion of CD25+ effector T cells. J Immunol 2006;176:7278-87.

6 Pasare C, Medzhitov R. Toll pathway-dependent blockade of CD4+CD25+ T cell-mediated suppression by dendritic cells. Science 2003;299:1033-6.

7 Peluso I, Fantini MC, Fina D, et al. IL-21 counteracts the regulatory T cell-mediated suppression of human CD4+ T lymphocytes. J Immunol 2007;178:732-9.

8 Valencia X, Stephens G, Goldbach-Mansky R, et al. TNF downmodulates the function of human CD4+CD25hi T-regulatory cells. Blood 2006;108:253-61.

9 Zhang J, Chen Y, Jia G, et al. FOXP3 -3279 and IVS9+459 polymorphisms are associated with genetic susceptibility to myasthenia gravis. Neurosci Lett 2013;534:274-8.

10 Gao L, Li K, Li F, et al. Polymorphisms in the FOXP3 gene in Han Chinese psoriasis patients. J Dermatol Sci 2010;57:51-6.

11 Park 0, Grishina I, Leung PS, et al. Analysis of the Foxp3/scurfin gene in Crohn's disease. Ann N Y Acad Sci 2005;1051:218-28.

12 Liston A, Lu LF, O'Carroll D, et al. Dicer-dependent microRNA pathway safeguards regulatory T cell function. J Exp Med 2008;205:1993-2004.

13 Chong MM, Rasmussen JP, Rudensky AY, et al. The RNAselll enzyme Drosha is critical in T cells for preventing lethal inflammatory disease. J Exp Med 2008;205:2005-17.

14 Bartel DP. MicroRNAs: genomics, biogenesis, mechanism, and function. Cell 2004;116:281-97.

15 O'Connell RM, Rao DS, Baltimore D. microRNA regulation of inflammatory responses. Annu Rev Immunol 2012;30:295-312.

16 Nakasa T, Miyaki S, Okubo A, et al. Expression of microRNA-146 in rheumatoid arthritis synovial tissue. Arthritis Rheum 2008;58:1284-92.

17 Stanczyk J, Pedrioli DM, Brentano F, et al. Altered expression of MicroRNA in synovial fibroblasts and synovial tissue in rheumatoid arthritis. Arthritis Rheum 2008;58:1001-9.

18 Kurowska-Stolarska M, Alivernini S, Ballantine LE, et al. MicroRNA-155 as a proinflammatory regulator in clinical and experimental arthritis. Proc Natl Acad Sci USA 2011;108:11193-8.

19 Pauley KM, Satoh M, Chan AL, et al. Upregulated miR-146a expression in peripheral blood mononuclear cells from rheumatoid arthritis patients. Arthritis Res Ther 2008;10:R101.

20 Li J, Wan Y, Guo Q, et al. Altered microRNA expression profile with miR-146a upregulation in CD4+ T cells from patients with rheumatoid arthritis. Arthritis Res Ther 2010;12:R81.

21 Nakasa T, Shibuya $H$, Nagata $Y$, et al. The inhibitory effect of microRNA-146a expression on bone destruction in collagen-induced arthritis. Arthritis Rheum 2011;63:1582-90.

22 Aletaha D, Neogi T, Silman AJ III, et al. 2010 Rheumatoid arthritis classification criteria: an American College of Rheumatology/European League Against Rheumatism collaborative initiative. Arthritis Rheum 2010;62:2569-81.

23 Arnett FC, Edworthy SM, Bloch DA, et al. The American Rheumatism Association 1987 revised criteria for the classification of rheumatoid arthritis. Arthritis Rheum 1988;31:315-24.

24 Ma X, Becker Buscaglia LE, Barker JR, et al. MicroRNAs in NF-kappaB signalling. J Mol Cell Biol 2011;3:159-66. 


\section{Basic and translational research}

25 Taganov KD, Boldin MP, Chang KJ, et al. NF-kappaB-dependent induction of microRNA miR-146, an inhibitor targeted to signalling proteins of innate immune responses. Proc Natl Acad Sci USA 2006;103:12481-6.

26 Tili E, Michaille JJ, Cimino A, et al. Modulation of miR-155 and miR-125b levels following lipopolysaccharide/TNF-alpha stimulation and their possible roles in regulating the response to endotoxin shock. J Immunol 2007;179:5082-9.

27 Lu LF, Boldin MP, Chaudhry A, et al. Function of miR-146a in controlling Treg cell-mediated regulation of Th1 responses. Cell 2010;142:914-29.

28 Kohlhaas S, Garden OA, Scudamore C, et al. Cutting edge: the Foxp3 target miR-155 contributes to the development of regulatory T cells. J Immunol 2009; 182:2578-82

29 Lu LF, Thai TH, Calado DP, et al. Foxp3-dependent microRNA155 confers competitive fitness to regulatory $T$ cells by targeting SOCS1 protein. Immunity 2009;30:80-91.

30 Thai TH, Calado DP, Casola $S$, et al. Regulation of the germinal center response by microRNA-155. Science 2007;316:604-8.

31 Rodriguez $A$, Vigorito $E$, Clare $S$, et al. Requirement of bic/microRNA-155 for normal immune function. Science 2007;316:608-11.

32 O'Connell RM, Rao DS, Chaudhuri AA, et al. Physiological and pathological roles for microRNAs in the immune system. Nat Rev Immunol 2010;10:111-22.
33 Hou J, Wang P, Lin L, et al. MicroRNA-146a feedback inhibits RIG-I-dependent Type I IFN production in macrophages by targeting TRAF6, IRAK1, and IRAK2. J Immunol 2009;183:2150-8.

34 Yang L, Boldin MP, Yu Y, et al. miR-146a controls the resolution of $\mathrm{T}$ cell responses in mice. J Exp Med 2012;209:1655-70.

35 Boldin MP, Taganov KD, Rao DS, et al. miR-146a is a significant brake on autoimmunity, myeloproliferation, and cancer in mice. J Exp Med 2011:208:1189-201.

36 O'Connell RM, Kahn D, Gibson WS, et al. MicroRNA-155 promotes autoimmune inflammation by enhancing inflammatory T cell development. Immunity 2010;33:607-19.

37 Tang Y, Luo X, Cui $\mathrm{H}$, et al. MicroRNA-146A contributes to abnormal activation of the type I interferon pathway in human lupus by targeting the key signalling proteins. Arthritis Rheum 2009;60:1065-75.

38 Walter GJ, Evans HG, Menon B, et al. Interaction with activated monocytes enhances cytokine expression and suppressive activity of human CD4+CD45ro +CD25+CD127(low) regulatory T cells. Arthritis Rheum 2013;65:627-38.

39 Sakaguchi S, Vignali DA, Rudensky AY, et al. The plasticity and stability of regulatory T cells. Nat Rev Immunol 2013;13:461-7.

40 Cvetanovich GL, Hafler DA. Human regulatory T cells in autoimmune diseases. Curr Opin Immunol 2010;22:753-60. 


\section{Decreased expression of miR-146a and} miR-155 contributes to an abnormal Treg phenotype in patients with rheumatoid arthritis

Qihui Zhou, Sonja Haupt, Johannes T Kreuzer, Ariane Hammitzsch, Fabian Proft, Carla Neumann, Jan Leipe, Matthias Witt, Hendrik Schulze-Koops and Alla Skapenko

Ann Rheum Dis 2015 74: 1265-1274 originally published online February 21,2014

doi: 10.1136/annrheumdis-2013-204377

Updated information and services can be found at:

http://ard.bmj.com/content/74/6/1265

\section{These include:}

Supplementary Supplementary material can be found at:

Material http://ard.bmj.com/content/suppl/2014/02/21/annrheumdis-2013-2043 77.DC1

References This article cites 40 articles, 17 of which you can access for free at: http://ard.bmj.com/content/74/6/1265\#BIBL

Email alerting Receive free email alerts when new articles cite this article. Sign up in the service box at the top right corner of the online article.

Topic
Collections

Articles on similar topics can be found in the following collections

Connective tissue disease (4253)

Degenerative joint disease (4641)

Immunology (including allergy) (5144)

Musculoskeletal syndromes (4951)

Rheumatoid arthritis (3258)

Inflammation (1251)

\section{Notes}

To request permissions go to:

http://group.bmj.com/group/rights-licensing/permissions

To order reprints go to:

http://journals.bmj.com/cgi/reprintform

To subscribe to BMJ go to:

http://group.bmj.com/subscribe/ 